\title{
Dominant Factors Affecting the Chromatographic Behaviour of Bile Acids
}

B. Natalini ${ }^{\bowtie}$, R. Sardella, E. Camaioni, S. Natalini, R. Pellicciari

Dipartimento di Chimica e Tecnologia del Farmaco, Università degli Studi di Perugia, Via del Liceo 1, 06123 Perugia, Italy;

E-Mail: natalini@chimfarm.unipg.it

\section{Erratum to: Chromatographia 64:343-349 \\ DOI 10.1365/s10337-006-0013-9}

The Publisher regrets that, unfortunately, the above article was published with a footnote which is not applicable. The paper was not presented at the 7th Symposium on the Practical Applications for the Analysis of Proteins, Nucleotides and Small Molecules, Montreal, Canada. 